\title{
Robustness and observability of rotating vortex lattices in an exciton-polariton condensate
}

\author{
Magnus O. Borgh, ${ }^{1}$ Guido Franchetti, ${ }^{2}$ Jonathan Keeling, ${ }^{3}$ and Natalia G. Berloff ${ }^{2}$ \\ ${ }^{1}$ School of Mathematics, University of Southampton, Southampton SO17 1BJ, United Kingdom \\ ${ }^{2}$ Department of Applied Mathematics and Theoretical Physics, University of Cambridge, Cambridge CB3 OWA, United Kingdom \\ ${ }^{3}$ SUPA, School of Physics and Astronomy, University of St. Andrews, St. Andrews KY16 9SS, United Kingdom
}

(Received 18 April 2012; published 9 July 2012)

\begin{abstract}
Exciton-polariton condensates display a variety of intriguing pattern-forming behaviors, particularly when confined in potential traps. It has previously been predicted that triangular lattices of vortices of the same sign will form spontaneously as the result of surface instabilities in a harmonic trap. However, natural disorder, deviation of the external potential from circular symmetry, or higher-order terms modifying the dynamical equations may all have detrimental effects and destabilize the circular trajectories of vortices. Here we address these issues by characterizing the robustness of the vortex lattice against disorder and deformations of the trapping potential. Since most experiments use time-integrated measurements, it would be hard to observe directly the rotating vortex lattices or distinguish them from vortex-free states. We suggest how these difficulties can be overcome and present an experimentally viable interference-imaging scheme that would allow the detection of rotating vortex lattices.
\end{abstract}

DOI: 10.1103/PhysRevB.86.035307

PACS number(s): 05.30.Jp, 03.75.Kk, 71.36.+c, 47.37.+q

\section{INTRODUCTION}

The experimental realization of Bose-Einstein condensates of quasiparticles in solid-state systems has provided a new example of macroscopically coherent quantum states of matter. Solid-state condensates exhibit striking fundamental differences from traditional quantum fluids such as atomic Bose gases and superfluid liquid helium. Much recent interest ${ }^{1-19}$ has been devoted to exciton-polaritons, which exist as normal modes of strongly coupled excitons and photons in semiconductor microcavities. Due to the short lifetime of the quasiparticles, these condensates exist in a dynamic balance between pumping and decay rather than in true thermal equilibrium. As such, these systems are capable of spatial and temporal pattern formation similar to that exhibited by lasers..$^{20,21}$

Quantized vortices ${ }^{3,7,9-14,17}$ and persistent currents ${ }^{9}$ as well as soliton dynamics ${ }^{16-18}$ have been observed in polariton condensates. Spatial patterns have been seen to arise spontaneously in both one ${ }^{8}$ and two ${ }^{15,19}$ dimensions. Intrinsic disorder in the material ${ }^{22}$ or deliberately designed potentials ${ }^{2}$ induce currents that may further facilitate symmetry breaking in the polariton system, leading to novel patterns. In particular, vortices have been seen to nucleate and get trapped because of the nonuniformities of the potential landscape. ${ }^{10,14}$

Most of the models that have been successfully and extensively used to describe pattern formation in polariton condensates are based on the complex Ginzburg-Landau equation (CGLE), which is also used in the theory of lasers and thus illustrates the similarities between the two systems. Such models have been applied to vortex nucleation and motion, ${ }^{17,23-26}$ spatial density modulations, ${ }^{15,27}$ solitons,,${ }^{16,17,23}$ and vortex lattices. ${ }^{28-31}$

Various different modifications of this model have been considered, such as including separate dynamics of the reservoir or modeling processes of thermal relaxation. Under the assumption of fast relaxation of the reservoir and small condensate densities these modifications all simplify, and the model can be written as

$$
\begin{aligned}
i \frac{\partial \psi}{\partial t}= & \frac{1}{2}\left[-\nabla^{2}+V(x, y)+|\psi|^{2}\right. \\
& \left.+i\left(\alpha(x, y)-\sigma|\psi|^{2}\right)\right] \psi,
\end{aligned}
$$

where $V(x, y)$ describes a trapping potential, $\alpha(x, y)$ is a (spatially varying) pump rate, and $\sigma$ is a nonlinearity which causes pumping to reduce as density increases; some such nonlinear process is clearly necessary for the system to be stable, and the form included is the simplest such nonlinearity. Linear losses are included in $\alpha$. Anticipating that we will consider a harmonically confined system, Eq. (1) is stated in oscillator units, measuring energy in units of $\hbar \omega$, length in units of the oscillator length $l=\sqrt{\hbar / m \omega}$, and time in units of $\omega^{-1}$, where $\omega$ is the oscillator frequency of the trapping potential, and $m$ is the effective mass of the polariton. The values assumed in the numerical simulations are discussed at the start of Sec. III.

The polarization degree of freedom of the polaritons provides additional possibilities in pattern and defect formation, ${ }^{32}$ such as allowing for spatially separate vortices of left and right polarization. ${ }^{7}$ Further possibilities for pattern formation arise in the presence of a magnetic field that favors circular polarization and therefore competes with the interactions, which favor equal densities of the polarization components, and also with any anisotropy resulting from strain fields induced by mechanical stress. The CGLE can be modified to include these effects, ${ }^{30}$ but in this paper we neglect polarization effects and consider only the spinless case described by Eq. (1).

In the absence of pumping and decay terms, the steady states of the CGLE are often well approximated by the stationary Thomas-Fermi solution. One rather surprising feature of the CGLE is that there exist many cases where the presence of pumping and decay significantly modifies the stable density profile. A particularly surprising case is that in a harmonic trap, the stationary state is unstable, and a rotating lattice is formed as the result of this instability. ${ }^{28}$ Spontaneously formed vortices have been observed in polariton condensates, ${ }^{3,7,11-13}$ 
but these have been isolated, stationary vortices, pinned by the disorder potential in the sample. The spontaneous formation of a regular, rotating vortex lattice ${ }^{28,30}$ has not been seen experimentally. The aim of this paper is therefore to study the robustness of such lattices and to discuss how one may overcome the difficulties that might arise in detecting a rotating vortex lattice.

We consider the robustness of vortex lattices with respect both to deformations of the circular harmonic trapping potential considered previously ${ }^{28,30}$ and to modifications of the CGLE, such as energy relaxation. We find that there is definite sensitivity to the trapping potential: both elliptical distortion and background disorder can destroy the regular vortex lattice and replace it with a chaotic regime, and we present numerical results for the critical distortion and disorder that destroy the lattice. This fragility with respect to the geometry of the trapping potential contrasts with the relative robustness of the vortex lattice to other terms that might be present in the CGLE, such as phenomenological approaches to the inclusion of relaxation processes.

Detection of moving vortices in a polariton condensate is nontrivial. Polariton condensates can be imaged directly using the photons escaping from the semiconductor microcavity. Each image requires that light be gathered over a nonnegligible period of time, and therefore any moving feature will tend to smear out. Hence rotating configurations would not be directly visible in time-integrated images. We show that these problems can be overcome by using a defocused homodyne imaging scheme, allowing stationary repeatable images to be observed, which fully characterize the vortex lattice.

The remainder of this paper is organized as follows. Section II discusses the instability of the stationary state, showing how this instability can be found analytically for weak pumping and decay. Section III then considers the vortex lattices that result from this linear instability, presenting results for elliptical trapping potentials and traps with additional disorder present. Section IV presents schemes for detecting rotating vortex lattices, and Sec. V summarizes these results.

\section{INSTABILITY OF THE NONROTATING STATE}

As commented above, the presence of pumping and decay destabilizes the stationary Thomas-Fermi potential in a circular trap, which in turn leads to the appearance of the vortex lattice. ${ }^{28}$ In this section we discuss how, for weak pumping and decay, this instability can be found analytically, providing some insight into how and why the stationary state becomes unstable. This section provides an outline and the main results of the analytical stability calculation; further technical details are given in Appendix A.

\section{A. Linear stability analysis without pumping and decay}

In order to find the leading-order effects of pumping and decay, one may linearize both in fluctuations and in the effects of pumping and decay; i.e., one considers first the density profile and fluctuations about this in the absence of pumping and decay and then determines how these are modified by nonzero $\alpha$ and $\sigma$. Using $\psi=\sqrt{\rho} e^{i \phi}$ and $V(x, y)=x^{2}+y^{2} \equiv$ $r^{2}$, one may rewrite Eq. (1) as a pair of real equations:

$$
\begin{aligned}
& \partial_{t} \rho+\nabla \cdot(\rho \vec{v})=(\alpha-\sigma \rho) \rho, \\
& 2 \partial_{t} \vec{v}+\nabla\left(\rho+r^{2}+|\vec{v}|^{2}\right)=0,
\end{aligned}
$$

where $\vec{v}=\nabla \phi$. In these equations, an approximation has already been made in neglecting the quantum pressure terms; this approximation is appropriate for sufficiently smooth density profiles (i.e., for clouds much larger than the healing length $\left.\xi_{\rho}=1 / \sqrt{\rho}\right)$. By substituting $\rho \rightarrow \rho+h(t), \vec{v} \rightarrow \vec{v}+$ $\vec{u}(t)$ into Eqs. (2) and linearizing in $\vec{u}, h$, the fluctuations obey

$$
\begin{gathered}
\partial_{t} h=-\nabla \cdot(\rho \vec{u}+h \vec{v})+(\alpha-2 \sigma \rho) h, \\
\partial_{t} \vec{u}=-\nabla(h / 2+\vec{v} \cdot \vec{u}) .
\end{gathered}
$$

In the absence of pumping and decay, the steady state is given by $\rho=\rho_{\mathrm{TF}} \equiv\left(\mu-r^{2}\right) \Theta\left(\mu-r^{2}\right)$, where $\Theta$ is the unit step function and $\vec{v}=0$, so the terms in Eq. (3) involving $\vec{v}$ vanish. In this case Eq. (3) can be reduced to the single equation

$$
\frac{d^{2} h}{d t^{2}}=\frac{1}{2 r} \frac{d}{d r}\left(r \rho \frac{d h}{d r}\right)+\frac{\rho}{2 r^{2}} \frac{d^{2}}{d \theta^{2}} h,
$$

which has eigenfunctions $i \partial_{t} h_{n, s}=\omega_{n, s} h_{n, s}$ labeled by angular momentum $h_{n, s}(r, \theta)=e^{i s \theta} h_{n, s}(r)$, where $h_{n, s}(r)$ are hypergeometric functions of the radial variable $r$ (see Appendix A 2). These eigenfunctions have frequencies

$$
\omega_{n, s}=\sqrt{s(1+2 n)+2 n(n+1)} .
$$

\section{B. Perturbative shift with pumping and decay}

We may now use solutions (4) and (5) as a basis on which to consider perturbatively the effects of pumping and decay. We will work here to first order in $\alpha$ and $\sigma$ in order to see the qualitative change introduced by pumping. This first-order treatment will predict an instability that already explains the behavior seen when all orders are considered in the numerical results presented in Ref. 28 and in Sec. III.

At first order, ${ }^{28}$ the steady state of Eq. (2) is given by $\rho=\rho_{\mathrm{TF}}$ and $\vec{v}=-\sigma \rho_{\mathrm{TF}} \vec{r} / 6$. Integrating the first equation of Eqs. (2) over the entire space gives $\mu=3 \alpha / 2 \sigma$. To find the change to the frequencies of the fluctuations, it is helpful to consider Eq. (3) in the form

$$
\left(\omega_{0 i}+\delta \omega_{i}\right)\left(\psi_{0 i}^{R}+\delta \psi_{i}^{R}\right)=i(\mathcal{L}+\alpha \delta \mathcal{L})\left(\psi_{0 i}^{R}+\delta \psi_{i}^{R}\right),
$$

where $\psi_{i}^{R}$ represent the right eigenfunctions of the matrix of differential operators $i \mathcal{L}$. Note that in this identification, the terms involving $\vec{v}$ in Eq. (3) should be considered part of $\delta \mathcal{L}$. The change to the eigenfrequency induced by pumping and decay is thus

$$
\delta \omega_{i}=i \alpha\left[\int d^{2} r \psi_{0 i}^{L} \delta \mathcal{L} \psi_{0 i}^{R}\right] /\left[\int d^{2} r \psi_{0 i}^{L} \psi_{0 i}^{R}\right],
$$

where $\psi_{i}^{L}$ indicate left eigenfunctions. Finding left and right eigenfunctions separately is necessary as the operator $i \mathcal{L}$ is not self-adjoint; further details are given in Appendix A 1.

\section{Instability of the stationary solution}

Using the hypergeometric solutions described above and following the outlined procedure (see Appendix A3 for 
details), one finds the shift

$$
\delta \omega_{i}=\frac{i \alpha}{4}\left[\frac{s^{2}-s(1+2 n)-2 n(n+1)}{\frac{1}{2} s^{2}+s(1+2 n)+2 n(n+1)}\right] .
$$

The crucial feature of this result is that for any $n$ there is always $s$ (for instance, $s \gg n$ ) such that this expression is positive. This implies that the eigenfrequencies acquire a positive imaginary part and the corresponding eigenmodes, which are those with large angular momentum, will therefore grow, leading to vortex nucleation (cf. atomic Bose-Einstein condensates where vortices are nucleated when external stirring causes unstable eigenmodes to arise ${ }^{33}$ ).

\section{Including phenomenological relaxation}

The same procedure may be repeated including various modifications to the CGLE. One such modification that may be easily incorporated is an energy-relaxation term, causing time evolution toward lower-energy states, i.e., $\partial_{t} \psi \rightarrow(1+$ $i \eta) \partial_{t} \psi$. The introduction of this term is common in literature on atomic Bose-Einstein condensates, where it models the atomic transfer between the condensate and the thermal cloud. These effects can be accurately modeled by a quantumBoltzmann equation describing the population dynamics of the quantum states. At lowest order of approximation this leads to the same term. ${ }^{34,35}$ Such terms have also been used in modeling polariton condensates. ${ }^{27,36,37}$ As described in Appendix A 4, including this model of relaxation at linear order changes the steady-state chemical potential to $\mu=3 \alpha /(2 \sigma+3 \eta)$, and the frequency shift becomes

$$
\delta \omega_{i}=\frac{i \alpha}{4}\left[\frac{\frac{2 \sigma}{2 \sigma+3 \eta} s^{2}-s(1+2 n)-2 n(n+1)}{\frac{1}{2} s^{2}+s(1+2 n)+2 n(n+1)}\right] .
$$

Thus this phenomenological model of relaxation, although driving the CGLE toward its low-energy states, is not sufficient to kill the instability; for any $n$ and any finite $\eta / \sigma$, there always exist modes (for instance, with $s \gg n \sigma / \eta$ ) which will become unstable.

The phenomenological relaxation term considered here is the simplest such term that can be included in the complex Ginzburg-Landau equation. More detailed models of relaxation, including also explicit dynamics of the reservoir, may remove the instability toward the rotating vortex lattice. The fact that the instability survives under the phenomenological relaxation considered here suggests that the instability may survive for small enough damping in more detailed models; however, this remains an open question.

\section{PROPERTIES OF THE ROTATING STATE}

The above stability analysis assumed an infinite pump spot; however, the same instability still exists as long as the pumping spot is larger than the condensate cloud size (given by $r_{\mathrm{TF}}=\sqrt{\mu}=\sqrt{3 \alpha / 2 \sigma}$ ). As found previously in Ref. 28 , the instability leads to a rotating vortex lattice in a circular pump spot. In this section we discuss the appearance of rotating states in cases where the trapping and pumping deviate from the ideal circular harmonic case considered in previous works. ${ }^{28,30}$ To discuss such cases, we present results of numerical simulations.
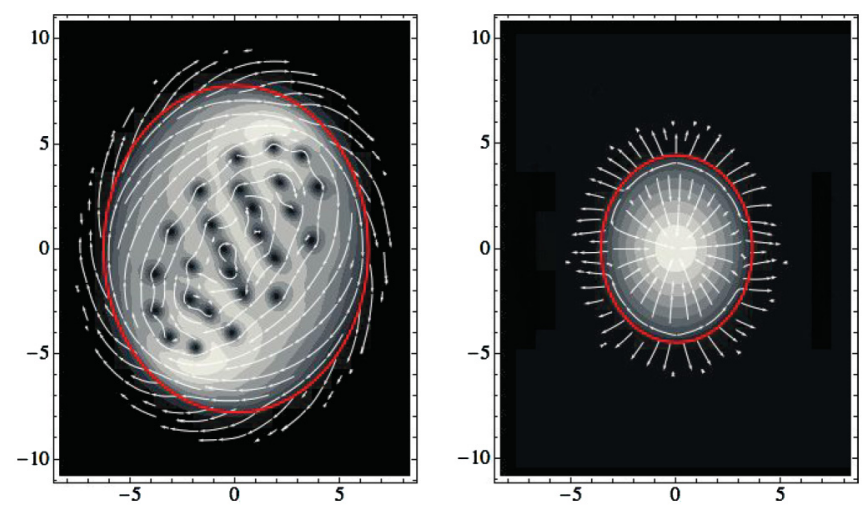

FIG. 1. (Color online) Density (grayscale) and superfluid streamlines (arrows) for different sizes of the pumping spot (red ellipse). (left) The surface instability leads to a spontaneously formed lattice with vortices moving on elliptical trajectories $(\delta=0.9, R=7)$. (right) The pumping spot is smaller than $r_{\mathrm{TF}}$ and the instability does not appear $(\delta=0.9, R=4)$.

In choosing values of the parameters $\alpha$ and $\sigma$ we follow the numbers used in Refs. 28 and 30. The parameter $\sigma$ may be directly related to the blueshift of the condensate when pumping at a given multiple of the threshold power. In this way $\sigma$ is estimated in Ref. 28 as $\sigma=0.3$. The dimensionless gain $\alpha$ is given by the background linewidth $\kappa$ and the pumping power $\gamma$ as $\alpha=2(\gamma-\kappa) / \hbar \omega$. Estimating the polariton lifetime at $\tau \simeq 5$ ps gives a linear decay rate $\kappa=\hbar / \tau=0.13 \mathrm{meV}$. To facilitate comparison with Refs. 28 and 30, we choose $\alpha=$ 4.4. In the stress trap constructed by Balili et al., ${ }^{2,38} \hbar \omega=$ $0.066 \mathrm{meV}$ for the harmonic confinement under the stressor, if the effective mass of the polariton is $m=7 \times 10^{-5} m_{e}$. For this value of $\hbar \omega$ our chosen $\alpha$ roughly corresponds to pumping at twice the threshold.

\section{A. Noncircular geometry}

Earlier considerations of spontaneously formed rotating vortex lattices in harmonic potentials ${ }^{28,30}$ have assumed circular symmetry. Here we lift this assumption by considering an elliptical geometry. For simplicity in illustrating the effects of elliptical geometry, we take both the trapping potential and the pumping spot in Eq. (1) to be described by a parameter $\delta$, such that ${ }^{39}$

$$
V(x, y)=\frac{x^{2}}{\delta^{2}}+\delta^{2} y^{2} .
$$

Using parameter values as detailed above, the pumping is taken to be constant inside the ellipse $x^{2} / \delta^{2}+\delta^{2} y^{2}=R^{2}$. The finite lifetime of the polaritons leads to a linear loss outside the pumping region. Since we are considering pumping at twice threshold, $\gamma=2 \kappa$, the value of $\alpha$ outside the pump spot is the same as inside but with opposite sign. As in the circular case, ${ }^{28}$ the surface instability will appear only if the pumping spot is sufficiently large, as illustrated in Fig. 1 for $\delta=0.9$. For our subsequent analysis, we keep a fixed $R=7$.

The movement of individual vortices in the lattice can be traced using a minimum-finding algorithm. The orbit is obtained by averaging the trajectory over a number of revolutions. In the regular vortex lattice, the individual vortices 

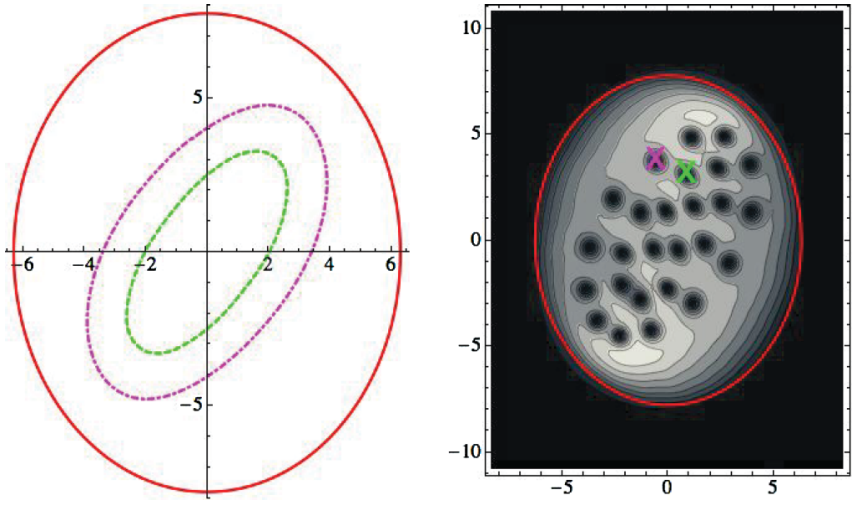

FIG. 2. (Color online) (left) The averaged orbits of two vortices (green dashed and magenta dash-dotted lines, respectively). The red solid ellipse marks the pumping spot. (right) Snapshot of the vortex lattice, with the tracked vortices marked by green and magenta crosses. $\delta=0.9$.

move on elliptic orbits, as shown in Fig. 2. Because the vortices have a definite sense of rotation, the motion is not symmetric around the major axis, and the resulting elliptic vortex lattice is tilted with respect to the trap.

In a sufficiently eccentric geometry, a steady array of vortices fails to form. We find a critical $\delta \sim 0.85$ for which some vortices move on elliptical orbits (Fig. 3, left). At $\delta=0.8$, the behavior is entirely chaotic, with vortices continually entering and leaving the cloud (Fig. 3, right), causing fragmentation of the condensate.

In the limit of extreme eccentricity, the condensate tries to establish a density profile of approximately uniform width in the transverse direction, leading to a less fragmented condensate at $\delta \lesssim 0.2$ (Fig. 4 ). The instability towards entrance of vortices is still present where the spot is widest: vortices entering and leaving the cloud cause transverse oscillations. As illustrated in Fig. 4, vortices of opposite signs may be present. The distribution of vortices of different sign may lead to a net effective pressure in the condensate pushing the perturbation along the length of the condensate until the pressure gradient
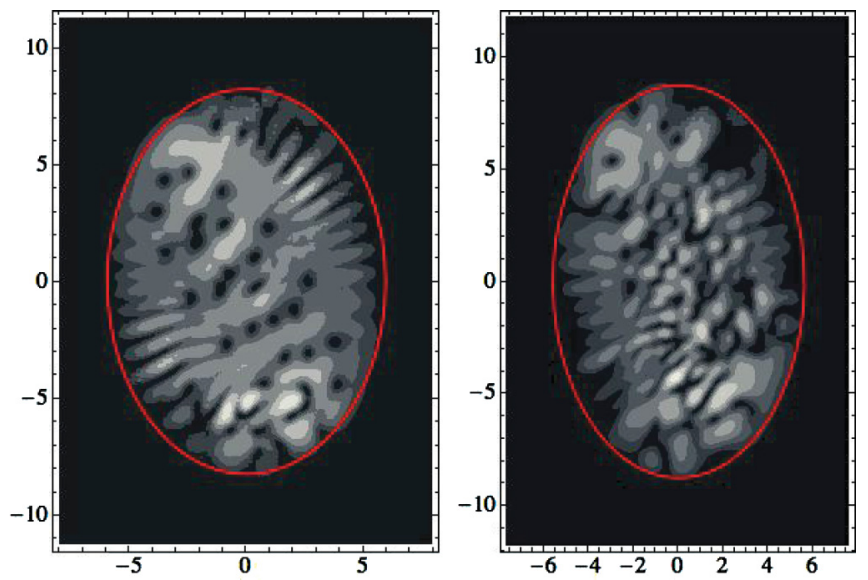

FIG. 3. (Color online) (left) $\delta=0.85$; the regular vortex lattice is broken up, with some vortices still on regular elliptic orbits. (right) $\delta=0.80$; as $\delta$ decreases, the rotating lattice gives way to a fragmented state with vortices continually entering and leaving the cloud.
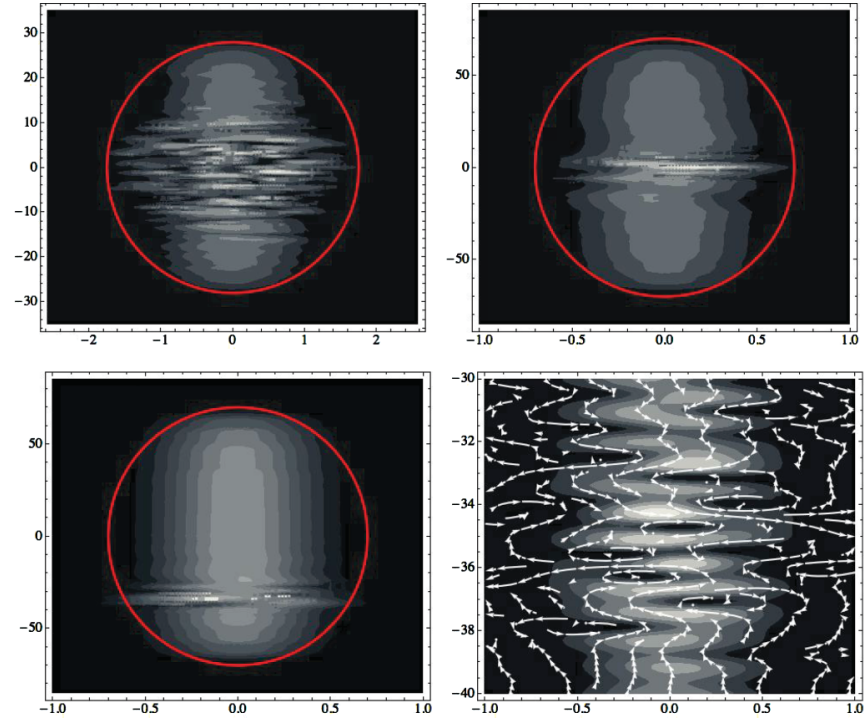

FIG. 4. (Color online) (top left) $\delta=0.25$. In a highly eccentric trap, the density tries to establish a profile of approximately uniform width along the length of the trap. Instability towards entrance of vortices leads to transverse oscillations. Note that the vertical axis has been rescaled so that the pumping spot appears circular in order to accommodate the extreme eccentricity. (top right) $\delta=0.1$. Extreme eccentricity suppresses fragmentation of the cloud. Transverse oscillations develop at the midpoint. (bottom left) Spontaneously entering vortices cause an effective pressure gradient, pushing the region of perturbations towards one end of the trap. The final position is shown. (bottom right) Vortices of both signs are present in the perturbed region. Arrows indicate circulation.

disappears. This effect is illustrated in Fig. 4 (bottom), showing the final position of the region of perturbations. Note that a true stationary state is not obtained: oscillations are still present.

We see that the formation of a regular vortex lattice is sensitive to the geometry of the system, in contrast to the robustness against refinements of the pumping model discussed in Secs. II B2 and IIIC. While a rotating vortex lattice will still form under small deviations from circular geometry, moderate eccentricity will prevent the lattice from forming.

The breakup of the regions of elliptical streamlines in a rotating classical flow is well known in fluid mechanics (e.g., see the review by Kerswell ${ }^{40}$ ). This "elliptical instability" is the linear instability of such a flow with the growth rates that scale with the strain rate. The nonlinear development of such instability leads to the breakdown of regular flow and may produce turbulence and small-scale disorder, similar to the outcome of the numerical simulations in our system. However, the elliptical instability of classical flows is essentially threedimensional, resulting from a parametric resonance of the normal modes with the straining field. Polariton condensates are invariably two-dimensional, so this type of instability is suppressed.

\section{B. Effects of disorder on the rotating state}

In the samples used in experiments disorder is always present. $^{22}$ Here we take this into account by including a disorder term in $V(x, y)$. This is modeled as a Gaussian random 

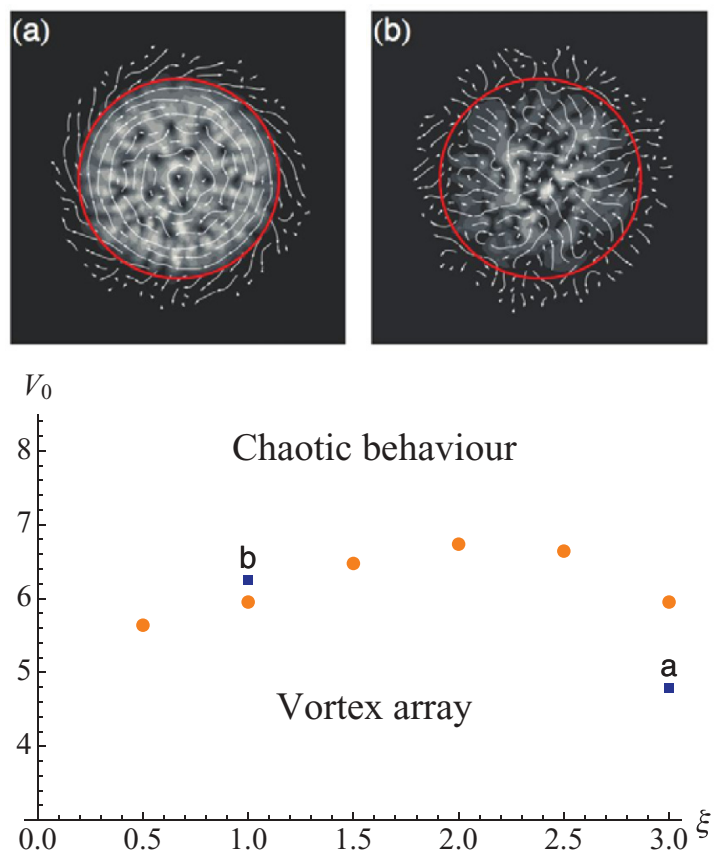

FIG. 5. (Color online) (top) The rotating vortex lattice forms also in the presence of weak disorder (a) but is destroyed by a disorder stronger than $V_{\text {crit }}$, leading to a chaotic state (b). (bottom) The boundary between vortex-lattice and chaotic regimes in the $\left(V_{0}, \xi\right)$ parameter space. The choices for $\left(V_{0}, \xi\right)$ leading to states (a) and (b) are indicated in the diagram. The vortex lattice is strongly sensitive to the amount of disorder, whereas the correlation length is of only marginal importance.

field with a real-space correlation function:

$$
\left\langle V_{\mathrm{dis}}(\vec{r}) V_{\mathrm{dis}}\left(\vec{r}^{\prime}\right)\right\rangle=V_{0}^{2} \exp \left(-\frac{\left(\vec{r}-\vec{r}^{\prime}\right)^{2}}{2 \xi^{2}}\right),
$$

where the amplitude $V_{0}$ is a measure of the strength of the disorder and $\xi$ is the correlation length. (Such a potential can be constructed by sampling the Fourier components of the disorder potential from a Gaussian distribution with a $k$-dependent variance; see Appendix B.)

Varying $V_{0}$ and $\xi$, we find that the formation of a vortex lattice is sensitive to the amount of disorder, with the lattice being destroyed for $V_{0}>V_{\text {crit }} \simeq 6.5$. When the disorder strength exceeds $V_{\text {crit }}$, the behavior is chaotic, and no stationary state forms. However, the critical amount of disorder is only weakly dependent on the disorder correlation length $\xi$, as illustrated by the nearly horizontal border in the $\left(V_{0}, \xi\right)$ diagram in Fig. 5. For the stress trap of Ref. 2 our estimate translates to a critical disorder $V_{\text {crit }} \simeq 0.4 \mathrm{meV}$, subject to uncertainties in estimates of experimental quantities as well as effects not accounted for in our model, such as relaxation mechanisms.

It is worth noting that $V_{\text {crit }}>\hbar \omega$ may give a false impression of a very large critical disorder strength. The relevant energy scale is given by the effective depth of the harmonic potential over the extent of the cloud, and this, in turn, is determined by the chemical potential $\mu \gg \hbar \omega$.

\section{Effects of other modifications to the CGLE model}

There are a number of other possible modifications to the simple CGLE model, Eq. (1), that could potentially destabilize a vortex lattice or prevent its formation: the presence of repulsive interactions between condensate particles and reservoir particles, the presence of higher-order nonlinear terms, or a finite gain linewidth leading to the appearance of superdiffusion in the governing equations. In this section we comment briefly on the possible consequences of considering these effects in the CGLE model.

Repulsive interactions with an excitonic reservoir. $\mathrm{Re}-$ pulsive interactions between reservoir polaritons and the condensate have been predicted theoretically ${ }^{41}$ and detected experimentally. ${ }^{8,42,43}$ For our geometry, such interactions may be modeled by adding a Gaussian potential to the center of the trap potential $V(x, y)$. The presence of this additional potential term does not prevent lattice formation but increases the probability of having a central, stationary vortex, as the reservoir provides a pinning site. The presence of Gaussian reservoir repulsion may further change the structure of the lattice: for example, a sharp and narrow Gaussian favors a square, rather than triangular, lattice.

Higher-order nonlinearities. One may consider higherorder corrections to polariton interactions in the form of a quintic nonlinearity in the CGLE. In some cases, inclusion of quintic terms in CGLE models is known to change the stability of the solutions. ${ }^{44}$ We have checked that the introduction of such terms in our model does not have any pronounced effect on vortex lattice formation.

Superdiffusion. Lasers emit particular transverse modes that depend on the detuning between the longitudinal modes of the reservoir (controlled by the resonator length) and the frequency at which gain is maximum. ${ }^{20}$ If such gain selection is relevant to the polariton condensates, the right-hand side of Eq. (1) acquires a term $i v\left(\nabla^{2}+\Delta\right)^{2} \psi$, where $\Delta$ is the detuning of the gain above the lowest transverse mode. Such mechanisms have not been extensively discussed in the context of polariton condensation, but the essence appears, for example, in Ref. 45. Our numerical simulations show that although the lattice survives for small values of $v$, the rings of vortices are shifted to the boundary of the condensate. The lattice disappears for $v>0.1$.

\section{DETECTING ROTATING VORTEX LATTICES}

We now discuss how time-integrated imaging techniques can be used to observe a nonstationary vortex lattice in experiments. Images are produced by gathering light escaping from the condensate over a period of time long enough that the movement of the vortices is non-negligible. We will construct a way of extracting an image that remains stationary during the measurement despite this movement. In practice, imaging is done by combining images from several realizations of the experiment. It is therefore necessary that the same image is produced in each realization. In the following, we discuss the relative merits of heterodyne and homodyne imaging techniques before combining the desirable features of both into an experimentally viable scheme described in Sec. IV C.

In the imaging schemes, we exploit the fact that a rotating vortex lattice generates sharp sidebands in the spectrum. ${ }^{30}$ 

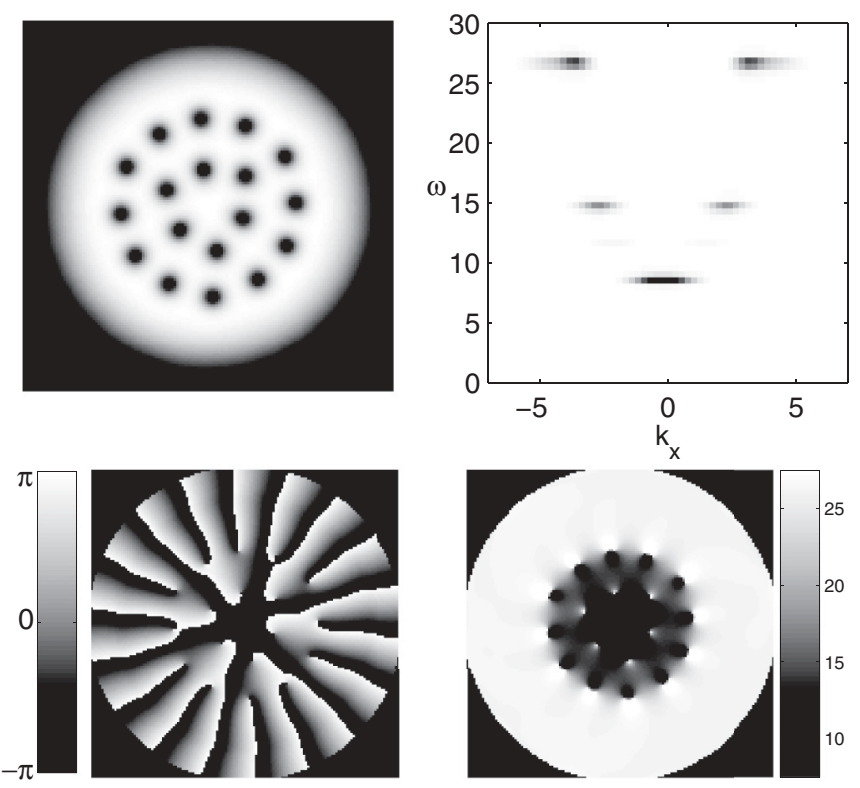

FIG. 6. Example of a vortex-lattice solution to Eq. (1). (top left) Density, showing the vortex lattice. (top right) The corresponding spectrum. Note the three bands corresponding to the regions of the condensate separated by the rings of vortices. (bottom left) Complex phase of the wave function and (bottom right) the time derivative of the phase. Note the separation into regions with an approximately constant time derivative corresponding to the bands in the spectrum.

A representative example is shown in Fig. 6. Each sideband corresponds exactly to one of the regions of the condensate separated by the rings of vortices. By interfering each region with light with matching frequency, the phase difference between light from the condensate and the interference beam will be constant in time, creating a fixed interference pattern, which can be integrated over time without smearing. (See Appendix $\mathrm{C}$ for technical details.)

\section{A. Heterodyne imaging}

We first consider a heterodyne interference scheme, in which the image of the condensate is made to interfere with a monochromatic reference beam without spatial structure, $\psi_{\text {ref }}=A_{0} \exp \left[-i\left(\theta_{0}+\omega_{0} t\right)\right]$. If $\omega_{0}$ is chosen to match one of the sidebands in the spectrum, the phase difference between the reference beam and condensate is independent of time in the region corresponding to the chosen sideband. Thus regions of constructive and destructive interference in this part of the image remain stationary.

Figure 7 shows interference images obtained from the example state of Fig. 6. The vortex lattice appears in the image as interference fringes, with the number of fringes corresponding to the number of vortices inside the stationary image.

This heterodyne scheme serves as a conceptually straightforward illustration of the type of image we would like to obtain. From an experimental point of view, however, the scheme is impracticable. A fundamental problem is that the initial phase $\theta_{0}$ is arbitrary. This means that while the interference fringes remain stationary within one measurement, there is no mechanism for ensuring that they appear in the same
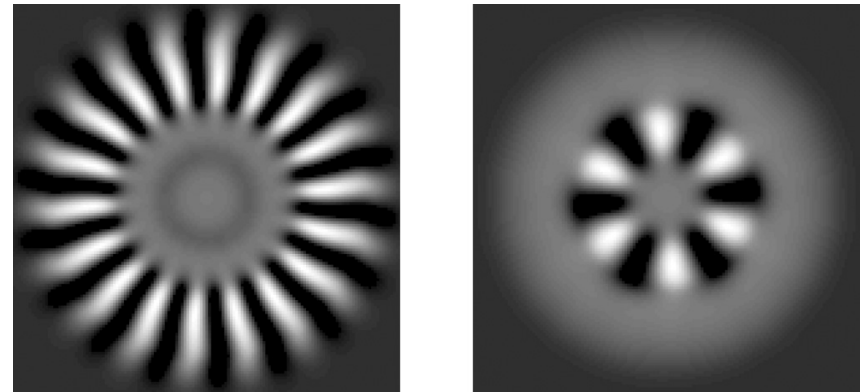

FIG. 7. Time-integrated interference image using the heterodyne scheme and the vortex lattice from Fig. 6. (left) $\omega_{0}=26.5$. (right) $\omega_{0}=14.5$.

place in any other realization (i.e., for locking the initial phase difference). This is crucial, as it means that while each image, in principle, will be clear, averaging over several realizations will cause the interference fringes to disappear. In addition to this fundamental problem, there may be practical difficulties in establishing a sufficiently stable phase reference at exactly the required frequency.

\section{B. Frequency-filtered homodyne imaging}

Since the heterodyne scheme has problems with the arbitrary phase reference, we next consider using a homodyne scheme to avoid this. The simplest homodyne scheme that would reveal information about a vortex lattice is one where an image of the condensate is filtered to a narrow frequency band and then made to interfere with a retroreflected and displaced copy of itself. This is a conceptually straightforward extension of well-established experimental techniques for detecting vortices in polariton condensates. ${ }^{3}$

Frequency filtering [Eq. (C3)] ensures that even after displacement, each part of the image interferes only with light of the same frequency, which is necessary to create a stationary interference pattern. The density and phase of the example lattice from Fig. 6 after filtering to $26.25 \leqslant \omega \leqslant 26.75$ are shown in Fig. 8.

The interference pattern created from Fig. 8 depends on the amount and direction of the displacement between the copies (Fig. 9). The images can be understood by considering how overlapping areas of the image in Fig. 8 and its retroreflected copy move in and out of phase as displacement is varied. In Fig. 9, this leads to destructive interference for small
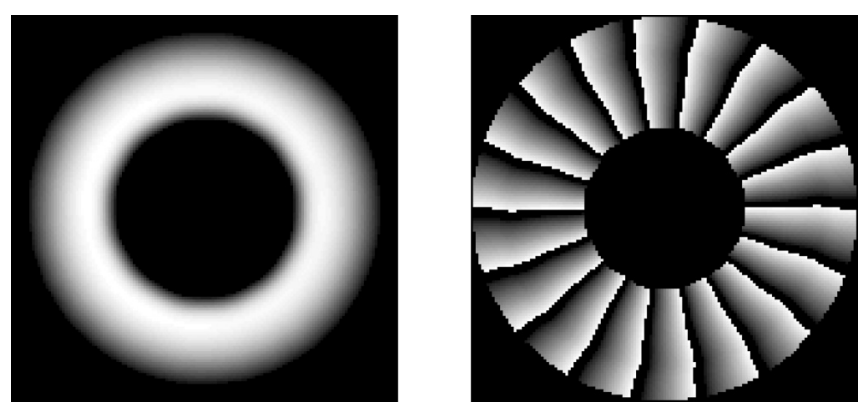

FIG. 8. Snapshot of (left) intensity and (right) phase after filtering $\psi$ from Fig. 6 to the band $26.25 \leqslant \omega \leqslant 26.75$. 

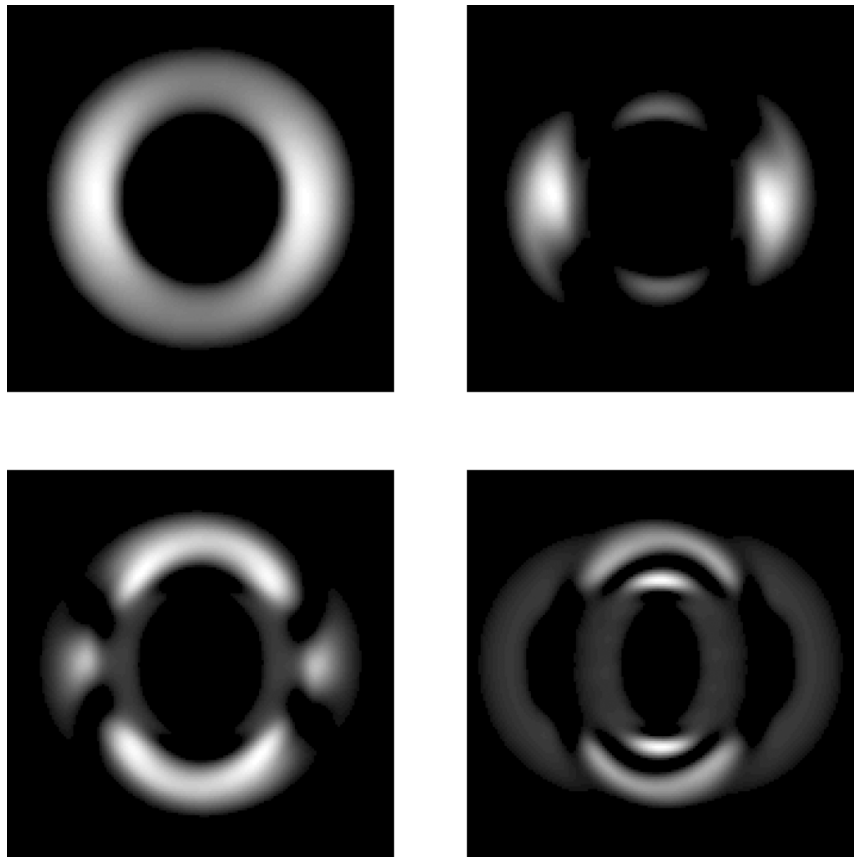

FIG. 9. Frequency-filtered homodyne scheme applied to the solution in Fig. 6 (the result of the filtering shown in Fig 8). From top left to bottom right, the displacement [see Eq. (C2)] is $x_{\mathrm{d}}=0.2$, $x_{\mathrm{d}}=0.5, x_{\mathrm{d}}=1.0$, and $x_{\mathrm{d}}=2.0$. (Here $y_{\mathrm{d}}=0$.)

displacement and to a complicated interference pattern as displacement is increased. ${ }^{46}$

Since the image is produced by interfering light from the condensate with itself, there is no arbitrary phase difference. The resulting image is therefore identical between realizations, and images from different realizations can be added without smearing. However, interpretation of the images is difficult. It is not straightforward to infer the vortex lattice from the interference image, let alone glean more detailed information about its structure.

\section{Defocused homodyne imaging}

We resolve the problems identified above by considering a defocused homodyne scheme constructed to combine the desirable features of the heterodyne and homodyne schemes. This will use a spatially uniform and nearly monochromatic reference beam, but with a mechanism for locking the arbitrary phase reference between realizations.

From the image of the condensate, light is taken from a reference point $\vec{r}_{0}$ and then defocused and made to interfere with the full image. Here we assume a reference point without spatial extent, corresponding to one grid point in the numerical simulation. In practice, the reference point would be a fixed spot small enough that the spatial variation of the phase across it is negligible. Optionally, a frequency filter [Eq. (C3)] may be applied as the first step of the scheme.

The scheme then amounts to forming the interference $I(\vec{r}, t)=\left|\psi(\vec{r}, t)+\psi\left(\vec{r}_{0}, t\right)\right|^{2}$. Choosing $\vec{r}_{0}$ to be in a ring where the time derivative of the phase is approximately constant (see Fig. 6) creates a reference beam with the frequency of a side band in the spectrum. Therefore, the interference image is qualitatively the same as that produced by the
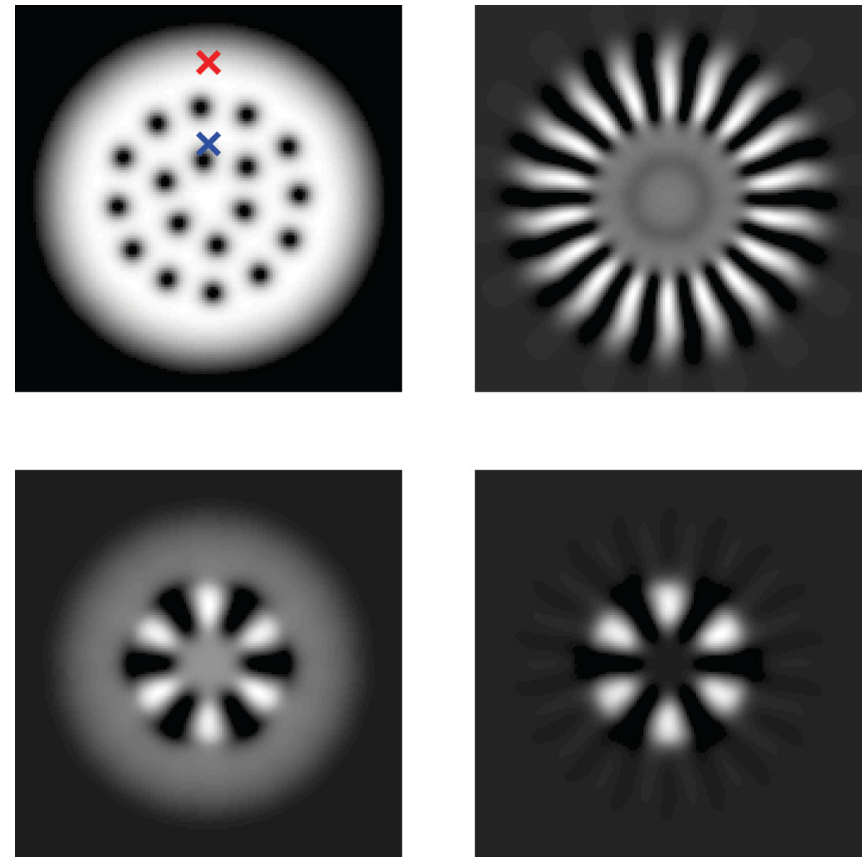

FIG. 10. (Color online) (top left) Snapshot of density, with crosses showing the points from which the reference is taken. (top right) Interference with the reference beam taken from the red cross, mapping out the interference fringes of all 18 vortices enclosed by that part of the condensate. (bottom left) Interference with light taken from the blue cross, giving six stationary interference fringes. (bottom right) Same as the bottom left panel, but with the image filtered to $14.15 \leqslant \omega \leqslant 14.75$ before the reference is extracted and allowed to interfere with the filtered image.

heterodyne scheme, but since the phase is now provided by the condensate itself, $\theta_{0}$ is no longer arbitrary. By construction, the interference at $\vec{r}_{0}$ is always constructive, thus locking the position of the interference fringes.

Figure 10 shows the defocused homodyne scheme applied to Fig. 6. The reference point $\vec{r}_{0}$ is indicated on the snapshot of the density. Selecting $\vec{r}_{0}$ between the vortex rings yields an interference image with six fringes corresponding to the inner ring of six vortices. Selecting $\vec{r}_{0}$ near the edge of the cloud reveals all 18 vortices.

The bottom panels of Fig. 10 show the effect of frequency filtering prior to applying the defocused homodyne scheme. Without filtering, interference fringes are mapped out the same way as in the heterodyne scheme. However, filtering may yield a clearer picture with better contrast.
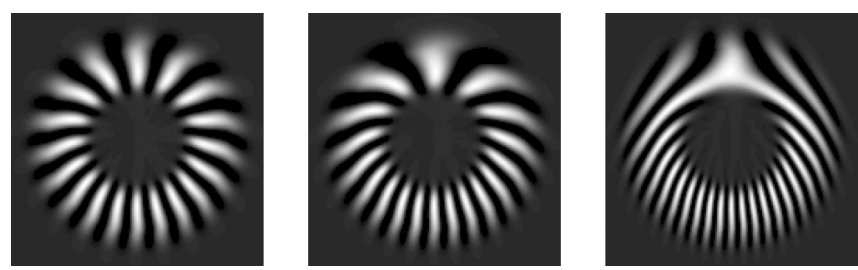

FIG. 11. Interference with light taken from the point indicated by the red cross in Fig. 10 after filtering to a narrow frequency band. The panels show the resulting interference image for different in-plane momenta in the $x$ direction. From left to right $k_{x}=1.0,2.0$, and 5.0. 

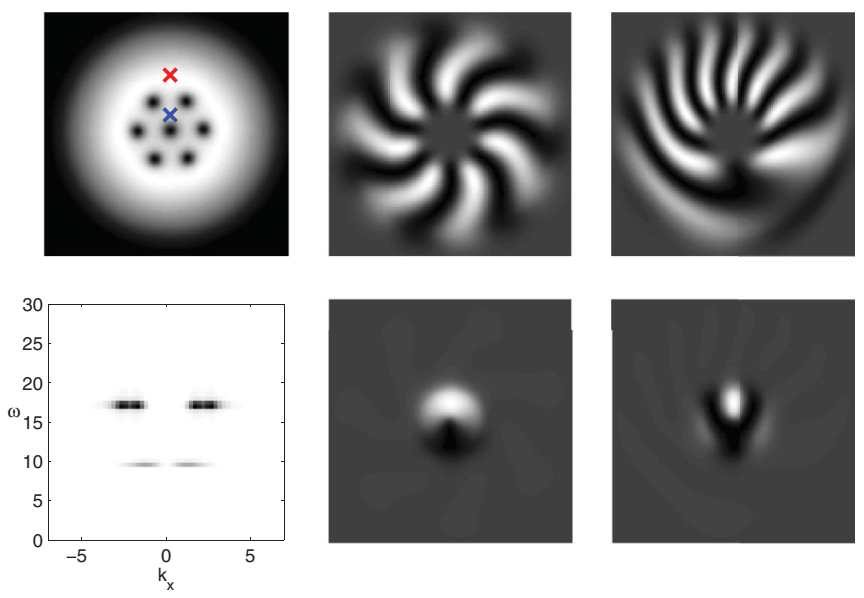

FIG. 12. (Color online) The top and bottom left panels show density and the corresponding spectrum. Crosses mark reference points for the filtered defocused homodyne scheme. The remaining panels show interference images corresponding to light taken from the red (top) and blue (bottom) crosses. From left to right in both rows, in-plane momentum in the $x$ direction $k_{x}=0, k_{x}=1$, and $k_{x}=2$.

A slight misalignment (intentional ${ }^{3}$ or accidental) in the interference optics may introduce a phase gradient in one of the pathways. In order to investigate whether the proposed scheme is robust against such misalignment we introduce a finite in-plane momentum $\vec{k}$ in the reference beam, such that $\psi_{\text {ref }}(\vec{r}, t)=\psi\left(\vec{r}_{0}, t\right) \exp (-i \vec{k} \cdot \vec{r})$. This results in a distortion of the interference fringes (Fig. 11). The distortion is small as long as $\vec{k}$ is not too large.

The distortion is more severe for smaller vortex lattices, as demonstrated in Fig. 12. Note that this lattice rotates in the opposite direction compared with Fig. 6 and that this is reflected in the distortion of the fringes in the presence of the phase gradient (cf. Fig. 11).

\section{CONCLUSIONS}

We have shown how the instability of high angular momentum modes leading to spontaneous formation of vortex lattices can be understood analytically. We have further addressed several difficulties involved in producing and detecting vortex lattices.

Our analytical treatment shows that the surface-mode instability is surprisingly robust against including a phenomenological model of relaxation in the simple models of pumping previously considered. Numerical analysis of the complex Ginsburg-Landau model reveals that both noncircular geometry and disorder may prevent formation of a vortex lattice, in contrast to its robustness against other modifications, such as relaxation, interactions with reservoir polaritons, and higher-order nonlinearities. Sufficient elliptic deformation or disorder results in a chaotic, nonstationary state. However, small deviations from circular symmetry as well as a small amount of disorder do allow a regular vortex lattice to form. The critical strength of disorder for $\alpha=4.4, \sigma=0.3$ is estimated at $\sim 6.5 \hbar \omega$, which corresponds to $\sim 0.4 \mathrm{meV}$ at twice-threshold pumping in the stress trap of Ref. 2.

We have further shown that spontaneously formed, rotating vortex lattices in a polariton condensate would be possible to detect using an experimentally feasible defocused homodyne scheme to generate stable interference images. By using a defocused homodyne scheme one can ensure that the same image is generated in each realization of the vortex lattice. Furthermore, the images contain direct information relating to the structure of the vortex lattice, allowing one, in principle, to establish the number of vortices and their arrangement within the lattice.

\section{ACKNOWLEDGMENTS}

M.O.B. acknowledges funding from the Leverhulme Trust. J.K. acknowledges funding from EPSRC Grants No. EP/G004714/2 and No. EP/I031014/1. G.F. acknowledges funding from Marie Curie Actions ESR grants. G.F. and N.G.B. acknowledge funding from EU CLERMONT4 235114.

\section{APPENDIX A: LINEAR STABILITY ANALYSIS}

This appendix provides further details of the analytic stability analysis presented in Sec. II. Section A 1 discusses the perturbation theory for Eq. (3), while Sec. A 2 gives the exact zeroth-order solutions of Eq. (4). Section A 3 then shows how the resulting integrals can be analytically evaluated, and Sec. A 4 discusses the effects of relaxation terms that may be added to the CGLE.

\section{Perturbation theory for Eq. (3)}

As briefly noted in Sec. II, the zeroth-order differential operator $i \mathcal{L}$ is not self-adjoint, and so evaluation of perturbation theory requires one to separately determine the left and right eigenfunctions of this operator. With such eigenfunctions identified, one may write the linear part of Eq. (6) as

$$
\delta \omega_{i} \psi_{0 i}^{R}+\omega_{0 i} \delta \psi_{i}^{R}=i \mathcal{L} \delta \psi_{i}^{R}+\alpha i \delta \mathcal{L} \psi_{0 i}^{R} .
$$

Following standard perturbation theory, one then writes

$$
\begin{aligned}
\delta \omega_{i} \int d^{2} r \psi_{0 i}^{L} \psi_{0 i}^{R}= & i \alpha \int d^{2} r \psi_{0 i}^{L} \delta \mathcal{L} \psi_{0 i}^{R} \\
& +\int d^{2} r \psi_{0 i}^{L}\left(i \mathcal{L}-\omega_{0 i}\right) \delta \psi_{i}^{R} .
\end{aligned}
$$

The last term will cancel as long as the operator $i \mathcal{L}$ has left eigenfunctions which satisfy

$$
I=\int d^{2} r \psi_{0 i}^{L} i \mathcal{L} \psi=\omega_{0 i} \int d^{2} r \psi_{0 i}^{L} \psi
$$

for all $\psi$.

The right eigenfunctions are given directly by solutions of Eq. (3). To find the corresponding left eigenfunctions, it is convenient to write out the components of the eigenfunctions as $\psi_{0 i}^{R}=\left(h_{0 i}^{R}, u_{r, 0 i}^{R}, u_{\theta, 0 i}^{R}\right)^{T}$ and $\psi_{0 i}^{L}=\left(h_{0 i}^{L}, u_{r, 0 i}^{L}, u_{\theta, 0 i}^{L}\right)$, where $u_{r}, u_{\theta}$ are radial and polar components of the velocity vector $\vec{u}$. The zeroth-order right eigenfunctions obey Eq. (3) with $\vec{v}=0, \alpha=0, \sigma=0$. This can be written out in components as

$$
\begin{gathered}
\omega_{0 i} h_{0 i}^{R}=-\frac{i}{r} \frac{d}{d r}\left(r \rho u_{r, 0 i}^{R}\right)+\frac{s}{r} \rho u_{\theta, 0 i}^{R}, \\
\omega_{0 i} u_{r, 0 i}^{R}=-\frac{i}{2} \frac{d h_{0 i}^{R}}{d r}, \quad \omega_{0 i} u_{\theta, 0 i}^{R}=\frac{s}{2 r} h_{0 i}^{R} .
\end{gathered}
$$


Writing out the components $\psi=\left(h, u_{r}, u_{\theta}\right)^{T}$, one has

$$
\begin{aligned}
I= & \int d^{2} r\left[-h_{0 i}^{L} \frac{i}{r} \frac{d}{d r}\left(r \rho u_{r}\right)+h_{0 i}^{L} \frac{s}{r} \rho u_{\theta}\right. \\
& \left.-u_{r, 0 i}^{L} \frac{i}{2} \frac{d h}{d r}+u_{\theta, 0 i}^{L} \frac{s}{2 r} h\right] .
\end{aligned}
$$

Integrating by parts [noting that the integral range is $0<r<$ $\sqrt{\mu}$ and that $r \rho(r)$ vanishes at the boundary], one may show that if $\left(h_{0 i}^{L}, u_{r, 0 i}^{L}, u_{\theta, 0 i}^{L}\right)=\left(h_{0 i}^{R *}, 2 \rho u_{r, 0 i}^{R *}, 2 \rho u_{\theta, 0 i}^{R *}\right)$, then

$$
I=\omega_{0 i} \int d^{2} r\left[h_{0 i}^{R *} h+2 \rho\left(u_{r, 0 i}^{R *} u_{r}+u_{\theta, 0 i}^{R *} u_{\theta}\right)\right],
$$

as required. Hence the above gives the relation between the left and right eigenfunctions.

\section{Exact zeroth-order solutions}

With the left eigenfunctions as defined above, the shift in frequency can be explicitly determined by evaluating Eq. (A1). The functions $u_{r, 0 i}^{R}, u_{\theta, 0 i}^{R}$ are given in terms of $h_{0 i}^{R}$ in Eq. (A2), and substituting into the equation for $h_{0 i}^{R}$ yields Eq. (4). For $\rho=$ $\rho_{\mathrm{TF}}$ and $h_{0 i}^{R}(r, \theta)=e^{i s \theta} h_{0 i}^{R}(r)$, this equation is hypergeometric. By restricting to solutions that remain bounded as $r^{2} \rightarrow \mu$, one finds the solutions to be the finite-order hypergeometric polynomials, with frequencies as given in Eq. (5) and wave functions

$$
h_{0 i}^{R}(r, \theta)=e^{i s \theta} r_{2}^{s} F_{1}\left(-n, s+1 ; n+s+1 ; r^{2}\right) .
$$

This simple form is the two-dimensional analog of the results given in three dimensions in Refs. 47 and 48. Note that the two-dimensional harmonic trap is not equivalent to the highly anisotropic pancake limit of the three-dimensional trap; the two-dimensional case presented here has a hard-wall boundary condition in the $z$ direction. As a result, the excitation spectrum given here is not the same as in Ref. 48.

\section{Evaluating overlaps for hypergeometric functions}

Using the results above, one finds that

$$
\begin{aligned}
\int & d^{2} r \psi_{0 i}^{L} \delta \mathcal{L} \psi_{0 i}^{R} \\
= & \int d^{2} r\left[(\alpha-2 \sigma \rho)\left|h_{0 i}^{R}\right|^{2}-\frac{h_{0 i}^{R *}}{r} \frac{d}{d r}\left(r h_{0 i}^{R} v\right)\right. \\
& \left.\quad-\frac{\rho}{2 \omega^{2}} \frac{d h_{0 i}^{R *}}{d r} \frac{d}{d r}\left(v \frac{d h_{0 i}^{R}}{d r}\right)-\frac{s^{2} v \rho h_{0 i}^{R *}}{2 \omega^{2} r^{2}} \frac{d h_{0 i}^{R}}{d r}\right] .
\end{aligned}
$$

By noting that $v(r)=|\vec{v}(r)|$ vanishes at the limits, one may integrate the second and third terms by parts. Using Eq. (4), one then finds that Eq. (A4) simplifies to

$$
\int d^{2} r \psi_{0 i}^{L} \delta \mathcal{L} \psi_{0 i}^{R}=\int d^{2} r\left[\alpha-2 \sigma\left(\mu-r^{2}\right)\right]\left|h_{0 i}^{R}\right|^{2} .
$$

Hence, making use of $\mu=3 \alpha / 2 \sigma$, Eq. (7) becomes $\delta \omega_{i}=$ $(i \alpha / 2)\left(3\left\langle r^{2}\right\rangle_{i} / \mu-2\right)$, where

$$
\left\langle r^{2}\right\rangle_{i}=\int d^{2} r r^{2}\left|h_{0 i}^{R}\right|^{2} / \int d^{2} r\left|h_{0 i}^{R}\right|^{2} .
$$

This integral can be found straightforwardly by using the identity

$$
0=\int_{0}^{\sqrt{\mu}} d r \frac{d}{d r}\left[\left(\rho r \frac{d h}{d r}\right)^{2}\right]=\int_{0}^{\sqrt{\mu}} d r \frac{d}{d r}\left[\frac{s^{2} \rho^{2}}{2}-\omega^{2} \rho r^{2}\right] h^{2}
$$

[making use of Eq. (4)]. Evaluating the second term and using the eigenvalues equation, one finds that $\left\langle r^{2}\right\rangle_{i}=\mu\left(s^{2}+\right.$ $\left.\omega_{i}^{2}\right) /\left(s^{2}+2 \omega_{i}^{2}\right)$. Using Eq. (5) for the eigenfrequencies, one recovers the result in Eq. (8).

\section{Stability of the generalized order parameter equation}

Including the phenomenological relaxation term discussed in Sec. II B2, the CGLE becomes

$$
\begin{aligned}
i \frac{\partial \psi}{\partial t}= & \frac{1}{2}\left[-\nabla^{2}+V(x, y)+|\psi|^{2}\right. \\
& \left.+i\left(\alpha(x, y)-\sigma|\psi|^{2}-2 i \eta \partial_{t}\right)\right] \psi,
\end{aligned}
$$

and the Madelung representation, Eqs. (2), becomes

$$
\begin{gathered}
\partial_{t} \rho+\nabla \cdot(\rho \vec{v})=\left(\alpha-\sigma \rho+2 \eta \partial_{t} \phi\right) \rho, \\
2 \partial_{t} \phi+\left(\rho+r^{2}+|\vec{v}|^{2}\right)=0 .
\end{gathered}
$$

The second of these equations has now been written explicitly for the phase, rather than for $\vec{v}=\nabla \phi$, since the phase now appears explicitly in the first equation. In keeping with the hydrodynamic approximation which neglects $\nabla^{2} \sqrt{\rho} / \sqrt{\rho}$, a similar term $\eta \partial_{t} \rho / \rho$ has also been neglected.

Since the second equation of Eqs. (A7) is unchanged, the stationary density profile remains as before $(\vec{v}$ is of first order in pumping and decay terms), with $\mu=-2 \partial_{t} \phi$. The continuity equation is, however, modified, so that integrating it over the entire space gives $\int 2 \pi r d r\left[\alpha-\sigma\left(\mu-r^{2}\right)-\eta \mu\right]\left(\mu-r^{2}\right)=$ 0 , yielding $\mu=3 \alpha /(2 \sigma+3 \eta)$.

The linearized fluctuation equations are complicated by the explicit appearance of $\phi$, so it is necessary to expand $\phi \rightarrow$ $\phi+\varphi(t)$; one finds

$$
\begin{aligned}
& \left(\begin{array}{cc}
1 & -2 \eta \rho \\
0 & 1
\end{array}\right) \partial_{t}\left(\begin{array}{l}
h \\
\varphi
\end{array}\right) \\
& =\left(\begin{array}{c}
-\nabla \cdot(\rho \vec{u}) \\
-h / 2
\end{array}\right)+\left(\begin{array}{c}
-\nabla \cdot(h \vec{v})+(\alpha-2 \sigma \rho-\eta \mu) h \\
-\vec{v} \cdot \vec{u}
\end{array}\right),
\end{aligned}
$$

where $\vec{u}=\nabla \varphi$. In the above, the time derivatives have been kept together on the left-hand side. One may then proceed by multiplying both sides by the inverse of the matrix appearing on the left and expanding to first order in the pumping and decay terms $\alpha, \sigma$, and $\eta$. The net result of this procedure is that the expression $(\alpha-2 \sigma \rho)$, which appeared in Eq. (A5), is now replaced by $(\alpha-2 \sigma \rho) \rightarrow(\alpha-2 \sigma \rho-\eta \mu-\eta \rho)$. Hence the frequency shift is given by $\delta \omega_{i}=(i / 2)[\alpha-2(\sigma+\eta) \mu+$ $\left.(2 \sigma+\eta)\left\langle r^{2}\right\rangle_{i}\right]$. Combining this with the values of $\mu$ and $\left\langle r^{2}\right\rangle_{i} / \mu$ given above gives Eq. (9).

\section{APPENDIX B: DISORDER POTENTIAL}

This appendix gives technical details for the inclusion of disorder discussed in Sec. III B. The disorder is included in 
the external potential by letting $V(\vec{r}) \rightarrow V(\vec{r})+V_{\text {dis }}(\vec{r})$, where $V_{\text {dis }}(\vec{r})$ is a Gaussian random field, constructed on the computational grid as a Fourier sum $V_{\mathrm{dis}}=\sum_{i, j}\left(a_{i j} / 2\right) \exp \left[i\left(k_{i} x+\right.\right.$ $\left.k_{j} y+\phi_{i j}\right)$, whose amplitudes $a_{i j}=a_{-i-j}$ are sampled from a Gaussian distribution with mean zero and standard deviation

$$
s_{i j}=v_{0} \exp \left[-l_{c}^{2}\left(k_{i}^{2}+k_{j}^{2}\right)\right] .
$$

The phase $\phi_{i j}$ is a random number in the interval $[0,2 \pi]$ with $\phi_{i, j}=-\phi_{-i,-j}$ and $k_{-i}=-k_{i}, k_{-j}=-k_{j}$. The quantities $v_{0}$ and $l_{c}$, defined for computational convenience, are related to the physical disorder strength $V_{0}$ and correlation length $\xi$ by noting that

$$
\left\langle V_{\mathrm{dis}}(\vec{r}) V_{\mathrm{dis}}\left(\vec{r}^{\prime}\right)\right\rangle \simeq \frac{v_{0}^{2}}{4(\Delta k)^{2}} \frac{\pi}{2 l_{c}^{2}} \exp \left[-\frac{\left(\vec{r}-\vec{r}^{\prime}\right)^{2}}{\left(8 l_{c}^{2}\right)}\right]
$$

and identifying $\xi=2 l_{c}$ and $V_{0}=v_{0} \sqrt{\pi / 2} /\left(2 l_{c} \Delta k\right)$, where $\Delta k$ is the numerical Fourier mode spacing. Equation (B2) follows from the fact that $\left\langle a_{i j} a_{u v} e^{i\left(\phi_{i j}+\phi_{u v}\right)}\right\rangle=\delta_{i, u} \delta_{j, v}\left\langle a_{i j}^{2} e^{2 \phi_{i j}}\right\rangle+$ $\delta_{i,-u} \delta_{j,-v}\left\langle a_{i j}^{2}\right\rangle=\delta_{i,-u} \delta_{j,-v} s_{i j}^{2}$ and replacing $k$-space sums by integrals.

\section{APPENDIX C: CONSTRUCTING INTERFERENCE IMAGES}

This appendix gives additional technical detail on the calculation of interference images. Vortex-lattice solutions are generated by propagating Eq. (1). The general principle for interference imaging of rotating vortex lattices discussed in Sec. IV does not, however, depend on the specific GinzburgLandau model. The dispersion spectrum of the condensate is found as the modulus squared of the three-dimensional Fourier transform of the condensate wave function sampled over a period of time:

$$
S(\omega, \mathbf{k})=\left|\int d^{2} r e^{-i \mathbf{k} \cdot \mathbf{r}} \int d t e^{-i \omega t} \psi(\mathbf{r}, t)\right|^{2} .
$$

The time-integrated interference images are found as the integral

$$
I(\vec{r})=\int_{T} d t\left|\psi\left(\vec{r}+\vec{r}_{\mathrm{d}}, t\right)+\psi_{\mathrm{ref}}\left(\vec{r}-\vec{r}_{\mathrm{d}}, t\right)\right|^{2},
$$

where the time $T$ is the time over which light is gathered in the measurement. Here we choose $T$ to correspond to a few revolutions of the vortex lattice. Since the interference image is stable, it is insensitive to the choice of $T$. The form of $\psi_{\text {ref }}$ is given by the choice of imaging method (Secs. IV A-IV C). The displacement $\vec{r}_{\mathrm{d}}$ is nonzero only for the homodyne scheme in Sec. IV B.

For the frequency-filtered homodyne and, optionally, the defocused homodyne schemes, the light gathered from the condensate is assumed to be passed through a band-pass filter that suppresses Fourier components outside the interval $\left[\omega_{\text {low }}, \omega_{\text {high }}\right]$, creating

$$
\psi_{\mathrm{filt}}(\vec{r}, t)=\frac{1}{2 \pi} \int_{\omega_{\mathrm{low}}}^{\omega_{\mathrm{high}}} d \omega\left[e^{i \omega t} \int_{-\infty}^{\infty} d t e^{-i \omega t} \psi(\vec{r}, t)\right],
$$

which is then used to create the interference image (C2).
${ }^{1}$ J. Kasprzak, M. Richard, S. Kundermann, A. Baas, P. Jeambrun, J. M. J. Keeling, F. M. Marchetti, M. H. Szymańska, R. André, J. L. Staehli, V. Savona, P. B. Littlewood, B. Deveaud, and L. S. Dang, Nature (London) 443, 409 (2006).

${ }^{2}$ R. Balili, V. Hartwell, D. Snoke, L. Pfeiffer, and K. West, Science 316, 1007 (2007).

${ }^{3}$ K. G. Lagoudakis, M. Wouters, M. Richard, A. Baas, I. Carusotto, R. André, L. S. Dang, and B. Devaud-Plédran, Nat. Phys. 4, 706 (2008).

${ }^{4}$ S. Utsunomiya, L. Tian, G. Roumpos, C. W. Lai, N. Kumada, T. Fujisawa, M. Kuwata-Gonokami, A. Löffler, S. Höfling, A. Forchel, and Y. Yamamoto, Nat. Phys. 4, 700 (2008).

${ }^{5}$ A. Amo, D. Sanvitto, F. P. Laussy, D. Ballarini, E. del Valle, M. D. Martin, A. Lemaître, J. Bloch, D. N. Krizhanovskii, M. S. Skolnick, C. Tejedor, and L. Viña, Nature (London) 457, 291 (2009).

${ }^{6}$ A. Amo, J. Lefrère, S. Pigeon, C. Adrados, C. Ciuti, I. Carusotto, R. Houdré, E. Giacobino, and A. Bramati, Nat. Phys. 5, 805 (2009).

${ }^{7}$ K. G. Lagoudakis, T. Ostatnický, A. V. Kavokin, Y. G. Rubo, R. André, and B. Deveaud-Plédran, Science 326, 974 (2009).

${ }^{8}$ E. Wertz, L. Ferrier, D. D. Solnyshkov, R. Johne, D. Sanvitto, A. Lemaître, I. Sagnes, R. Grousson, A. V. Kavokin, P. Senellart, G. Malpuech, and J. Bloch, Nat. Phys. 6, 860 (2010).

${ }^{9}$ D. Sanvitto, F. M. Marchetti, M. H. Szymańska, G. Tosi, M. Baudisch, F. P. Laussy, D. N. Krizhanovskii, M. S. Skolnick, L. Marrucci, A. Lemaître, J. Bloch, C. Tejedor, and L. Viña, Nat. Phys. 6, 527 (2010).
${ }^{10}$ G. Nardin, K. G. Lagoudakis, B. Pietka, F. Morier-Genoud, Y. Léger, and B. Deveaud-Plédran, Phys. Rev. B 82, 073303 (2010).

${ }^{11}$ G. Roumpos, M. D. Fraser, A. Löffler, S. Höfling, A. Forchel, and Y. Yamamoto, Nat. Phys. 7, 129 (2010).

${ }^{12}$ G. Tosi, F. M. Marchetti, D. Sanvitto, C. Antón, M. H. Szymańska, A. Berceanu, C. Tejedor, L. Marrucci, A. Lemaître, J. Bloch, and L. Viña, Phys. Rev. Lett. 107, 036401 (2011).

${ }^{13}$ K. G. Lagoudakis, F. Manni, B. Pietka, M. Wouters, T. C. H. Liew, V. Savona, A. V. Kavokin, R. André, and B. Deveaud-Plédran, Phys. Rev. Lett. 106, 115301 (2011).

${ }^{14}$ G. Nardin, G. Grosso, Y. Léger, B. Pietka, F. Morier-Genoud, and B. Deveaud-Plédran, Nat. Phys. 7, 635 (2011).

${ }^{15}$ F. Manni, K. G. Lagoudakis, T. C. H. Liew, R. André, and B. Deveaud-Plédran, Phys. Rev. Lett. 107, 106401 (2011).

${ }^{16}$ A. Amo, S. Pigeon, D. Sanvitto, V. G. Sala, R. Hivet, I. Carusotto, F. Pisanello, G. Leménager, R. Houdré, E. Giacobino, C. Ciuti, and A. Bramati, Science 332, 1167 (2011).

${ }^{17}$ G. Grosso, G. Nardin, F. Morier-Genoud, Y. Léger, and B. DeveaudPlédran, Phys. Rev. Lett. 107, 245301 (2011).

${ }^{18}$ M. Sich, D. N. Krizhanovskii, M. S. Skolnick, A. V. Gorbach, R. Hartley, D. V. Skryabin, E. A. Cerda-Méndez, K. Biermann, R. Hey, and P. V. Santos, Nat. Photonics 6, 50 (2012).

${ }^{19}$ G. Tosi, G. Christmann, N. G. Berloff, P. Tsotsis, T. Gao, Z. Hatzopoulos, P. G. Savvidis, and J. J. Baumberg, Nat. Phys. 8, 190 (2012).

${ }^{20}$ F. Arecchi, S. Boccaletti, and P. Ramazza, Phys. Rep. 318, 1 (1999). 
${ }^{21}$ K. Staliunas and V. J. Sanchez-Morcillo, Transverse Patterns in Nonlinear Optical Resonators, Springer Tracts in Modern Physics Vol. 183 (Springer, Berlin, 2003).

${ }^{22}$ V. Savona, J. Phys. Condens. Matter 19, 295208 (2007).

${ }^{23}$ S. Pigeon, I. Carusotto, and C. Ciuti, Phys. Rev. B 83, 144513 (2011).

${ }^{24}$ M. D. Fraser, G. Roumpos, and Y. Yamamoto, New J. Phys. 11, 113048 (2009).

${ }^{25}$ H. Flayac, G. Pavlovic, M. A. Kaliteevski, and I. A. Shelykh, Phys. Rev. B 85, 075312 (2012).

${ }^{26}$ F. M. Marchetti, M. H. Szymańska, C. Tejedor, and D. M. Whittaker, Phys. Rev. Lett. 105, 063902 (2010).

${ }^{27}$ M. Wouters, T. C. H. Liew, and V. Savona, Phys. Rev. B 82, 245315 (2010).

${ }^{28}$ J. Keeling and N. G. Berloff, Phys. Rev. Lett. 100, 250401 (2008).

${ }^{29}$ T. C. H. Liew, Y. G. Rubo, and A. V. Kavokin, Phys. Rev. Lett. 101, 187401 (2008).

${ }^{30}$ M. O. Borgh, J. Keeling, and N. G. Berloff, Phys. Rev. B 81, 235302 (2010).

${ }^{31}$ A. V. Gorbach, R. Hartley, and D. V. Skryabin, Phys. Rev. Lett. 104, 213903 (2010).

${ }^{32}$ I. A. Shelykh, A. V. Kavokin, Y. G. Rubo, T. C. H. Liew, and G. Malpuech, Semicond. Sci. Technol. 25, 013001 (2010).

${ }^{33}$ S. Sinha and Y. Castin, Phys. Rev. Lett. 87, 190402 (2001).

${ }^{34}$ A. A. Penckwitt, R. J. Ballagh, and C. W. Gardiner, Phys. Rev. Lett. 89, 260402 (2002).
${ }^{35}$ C. W. Gardiner, P. Zoller, R. J. Ballagh, and M. J. Davis, Phys. Rev. Lett. 79, 1793 (1997)

${ }^{36}$ M. Wouters and I. Carusotto, Phys. Rev. Lett. 105, 020602 (2010).

${ }^{37}$ M. Wouters and V. Savona, arXiv:1007.5453.

${ }^{38}$ R. B. Balili, D. W. Snoke, L. Pfeiffer, and K. West, Appl. Phys. Lett. 88, 031110 (2006)

${ }^{39}$ The deformation $\delta$ is related to the eccentricity of the ellipse as $\varepsilon=\sqrt{1-1 / \delta^{4}}$.

${ }^{40}$ R. R. Kerswell, Annu. Rev. Fluid Mech. 34, 83 (2002).

${ }^{41}$ M. Wouters and I. Carusotto, Phys. Rev. Lett. 99, 140402 (2007).

${ }^{42}$ L. Ferrier, E. Wertz, R. Johne, D. D. Solnyshkov, P. Senellart, I. Sagnes, A. Lemaître, G. Malpuech, and J. Bloch, Phys. Rev. Lett. 106, 126401 (2011).

${ }^{43}$ G. Christmann, G. Tosi, N. G. Berloff, P. Tsotsis, P. S. Eldridge, Z. Hatzopoulos, P. G. Savvidis, and J. J. Baumberg, Phys. Rev. B 85, 235303 (2012).

${ }^{44}$ I. S. Aranson and L. Kramer, Rev. Mod. Phys. 74, 99 (2002).

${ }^{45}$ T. D. Doan, H. T. Cao, D. B. Tran Thoai, and H. Haug, Phys. Rev. B 74, 115316 (2006).

${ }^{46}$ In Fig. 9 an even number of vortices are enclosed by the filtered image. If an odd number of vortices were enclosed, regions of constructive and destructive interference would be interchanged.

${ }^{47}$ S. Stringari, Phys. Rev. Lett. 77, 2360 (1996).

${ }^{48}$ S. Stringari, Phys. Rev. A 58, 2385 (1998). 